\title{
Synthesis of Liquid Crystalline Oligomeric Polyoxetanes with Two Mesogenic Groups in the Repeating Unit
}

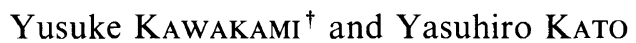 \\ Graduate School of Materials Science, Japan Advanced Institute of Science and Technology, \\ Asahidai 1-1, Tatsunokuchi, Ishikawa 923-12, Japan
}

(Received February 17, 1997)

\begin{abstract}
KEY WORDS Oxetane / Cationic Ring-Opening Polymerization / Polyether / Mesogen / Thermal Analysis / Liquid Crystalline Polymer / Nematic / Smectic /
\end{abstract}

It is of interest to control the liquid crystalline state by the introduction of second components in the polymer backbone. We reported an example in which a second substituent was introduced to the same carbon atom, to which mesogenic group is connected, of the repeating unit of polyoxetane. By introduction of siloxane linkage in the substituent, liquid crystalline temperature range became wider. ${ }^{1}$ Much interest has been also paid to the properties of side-chain liquid crystalline polymers containing two or more different mesogenic groups. These polymers have been mainly obtained by hydrosilylation reaction by the hydrosilyl group of poly(hydromethylsiloxane). ${ }^{2-4}$ By this method, mesogenic groups were randomly introduced. Introduction of a second component by copolymerization is an interesting way to introduce a variety of mesogenic groups to vinyl polymers. ${ }^{5-9}$ However, it is quite difficult to introduce the mesogenic groups at specific position of the repeating unit because the mesogens are introduced on the basis of the monomer reactivity ratio. Examples of liquid crystalline block copolymer are recently reported. ${ }^{10}$ In this case, there is the possibility for the two components to micro-phase separate.

There have been only few examples in with plural numbers of mesogenic groups introduced in the repeating unit of liquid crystalline polymers. ${ }^{5,11-13}$ Since these polymers contain high concentrations of or variety of mesogenic groups, it is of interest to study the liquid crystalline phase exhibited by these polymers. Mesogens were usually introduced after polymerization reaction because of the difficulty in the synthesis of the corresponding monomers. From the standpoint of the quantitative introduction of a second mesogen to specific position to the first mesogen, polymerization of monomers with two mesogenic groups is the preferred method. By this method, a side-chain liquid crystalline polymer without micro-phase separation of two components is obtained.

A typical polymer in this study has two cyanobiphenyl groups as substituents in the repeating unit. Various combinations of mesogenic and spacer groups were used in order to study the effects of polymer structure on the phase and the temperature range of the liquid crystalline state.

\footnotetext{
† To whom correspondence should be addressed.
}

\section{EXPERIMENTAL}

General

${ }^{1} \mathrm{H}$ and ${ }^{13} \mathrm{C}$ NMR spectra were recorded on a Varian $300 \mathrm{MHz}{ }^{1} \mathrm{H}$ NMR $\left(75 \mathrm{MHz}\right.$ for $\left.{ }^{13} \mathrm{C}\right)$ spectrometer Model Gemini 300. GPC analyses were carried out on a JASCO GPC Model HLC 980 equipped with Shodex gels KF801 (exclusion molecular weight, polystyrene $1.5 \times 10^{3}$ ) and KF804 (exclusion molecular weight, polystyrene $4 \times 10^{5}$ ) using tetrahydrofuran. DSC analyses were carried out on a SEIKO thermal analysis system Model SSC 5200 equipped with DSC 120 at a heating rate of $5^{\circ} \mathrm{C} \mathrm{min}^{-1}$. Optical polarization micrographs were taken on a Nikon optical polarization micrograph Model OPTIPHOTO2-POL equipped with a Mettler thermal analysis system Model FP900 with FP82HT hot stage and FP 90 controller. Pictures were taken at a little lower temperature than the peak after annealing in cooling from isotropic state at appropriate intervals.

\section{Synthesis}

The synthesis of the monomers is quite similar to that already reported. ${ }^{14}$ The synthetic route is shown in Scheme 1.

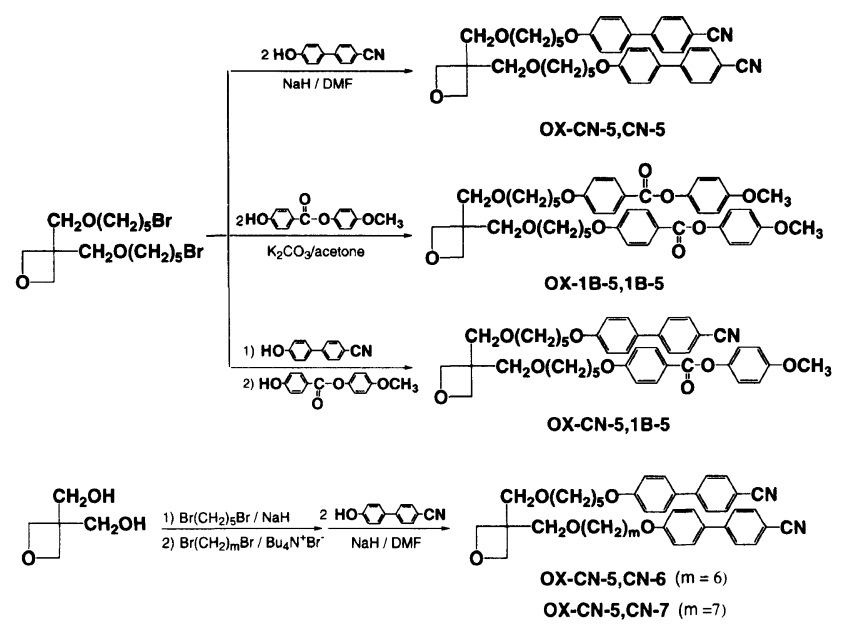

Scheme 1. Synthetic routes to monomers.

The names of the monomers are abbreviated by the combination of oxetane $(\mathrm{OX})$; the mesogen $(\mathrm{CN}$, cyanobiphenyl; 1B, methoxyphenyl benzoate), and length 
of spacer ( $m, n$, number of methylene unit in the spacers). For example, OX-CN-5,1B-5 indicates the monomer with cyanobiphenyl and 5 methylenes as the first mesogen and the spacer, and methoxyphenyl benzoate connected through 5 methylenes as the second mesogenic group. Polymers are abbreviated by adding $P$ to the abbreviated name of the monomer.

Protons of oxetane and mesogenic groups, and carbon atom of the polymers are indicated in Figure 1.

Assignments of the proton and carbon signals of the polymers were made similarly to the reported results. ${ }^{1,14,15}$

3,3-Bis(5-bromopentyloxymethyl)oxetane. To a mixture of $50 \mathrm{wt} \%$ aqueous $\mathrm{NaOH}(200 \mathrm{~mL})$ and hexane $(200 \mathrm{~mL})$, and tetrabutylammonium bromide $(0.32 \mathrm{~g}$, $5 \mathrm{mmol}$ ) were added 3,3-bis(hydroxymethyl)oxetane $(11.8 \mathrm{~g}, 100 \mathrm{mmol})^{16}$ and 1,5-dibromopentane (230 g, $1 \mathrm{~mol}$ ). The mixture was refluxed for 3 days. After organic layer was separated, the aqueous layer was extracted with hexane $(200 \mathrm{~mL} \times 2)$. The combined organic layer was washed with $1 \mathrm{wt} \%$ aqueous $\mathrm{HCl}(300 \mathrm{~mL})$ and water $(300 \mathrm{~mL})$ successively, and dried over anhydrous magnesium sulfate. Removal of the solvent and low boiling materials under reduced pressure followed by silica gel column chromatography (eluent, hexane:ethyl acetate $=3: 1$ ) gave a colorless liquid as the product.

Yield $52 \% . R_{\mathrm{f}}=0.55$ (hexane : ethyl acetate $=2: 1$ ).

${ }^{1} \mathrm{H}$ chemical shifts: $1.47-1.88\left(\mathrm{~m}, 12 \mathrm{H}, \mathrm{OCH}_{2} \mathrm{CH}_{2}-\right.$ $\left.\mathrm{CH}_{2} \mathrm{CH}_{2} \mathrm{CH}_{2} \mathrm{O}\right), 3.41\left(\mathrm{t}, 4 \mathrm{H}, \mathrm{J}=6.0 \mathrm{~Hz}, \mathrm{CH}_{2} \mathrm{Br}\right), 3.47(\mathrm{t}$, $\left.4 \mathrm{H}, J=6.0 \mathrm{~Hz}, \mathrm{CCH}_{2} \mathrm{OCH}_{2}\right), 3.61\left(\mathrm{~s}, 4 \mathrm{H}, \mathrm{CCH}_{2} \mathrm{O}\right), 4.46$ (s, 4H, Ha, $H \mathrm{~b})$.

3-\{(5-Bromopentyloxy)methyl $\}-3-($ hydroxymethyl $)$ oxetane. Excess 1,5-dibromopentane (115 g, $500 \mathrm{mmol})$ was reacted with 3,3-bis(hydroxymethyl)oxetane (11.8 g, $100 \mathrm{mmol})$ beforehand treated with sodium hydride $(4.2 \mathrm{~g}, 105 \mathrm{mmol})$ in dimethylformamide (DMF) (150 $\mathrm{mL}$ ) at room temperature for overnight. Ordinary work up followed by removal of excess 1,5-dibromopentane
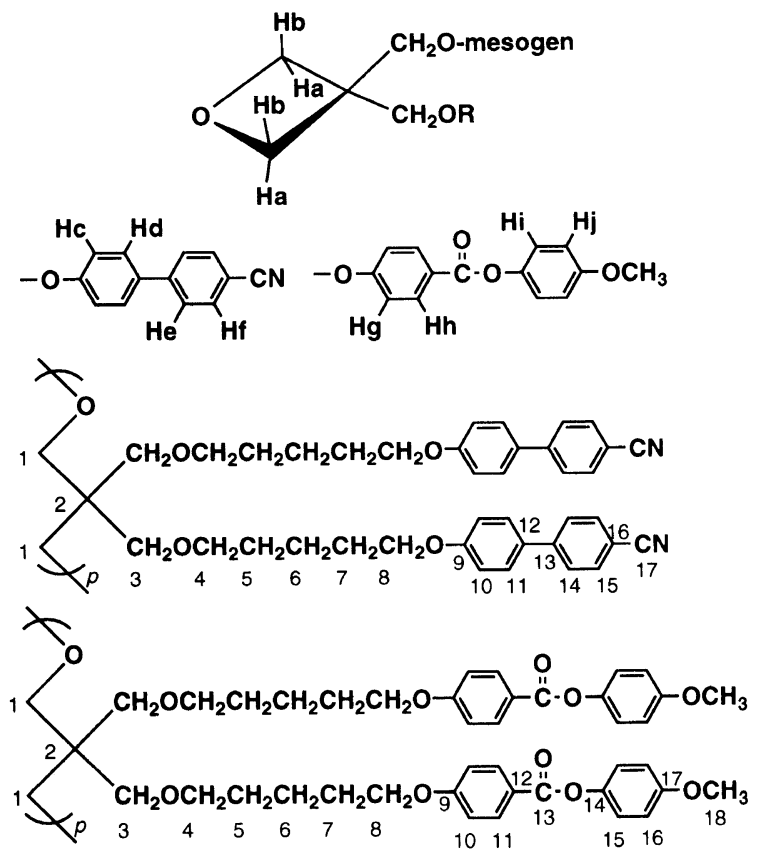

Figure 1. Identification of proton and carbon atoms of monomers and polymers. under reduced pressure gave an oil. Purification by silica gel column chromatography (eluent, hexane : ethyl acetate $=3: 1$ ) gave a colorless product as a liquid.

Yield $22 \% . R_{\mathrm{f}}=0.35$ (hexane : ethyl acetate $=1: 1$ )

${ }^{1} \mathrm{H}$ chemical shifts: $1.45-1.87\left(\mathrm{~m}, 6 \mathrm{H}, \mathrm{OCH}_{2} \mathrm{CH}_{2}\right.$ $\left.\mathrm{CH}_{2} \mathrm{CH}_{2} \mathrm{CH}_{2} \mathrm{O}\right), 3.41\left(\mathrm{t}, 2 \mathrm{H}, \mathrm{J}=6.0 \mathrm{~Hz}, \mathrm{CH}_{2} \mathrm{Br}\right), 3.49(\mathrm{t}$, $\left.2 \mathrm{H}, \mathrm{J}=6.0 \mathrm{~Hz}, \mathrm{CCH}_{2} \mathrm{OCH}_{2}\right), 3.78\left(\mathrm{~s}, 2 \mathrm{H}, \mathrm{CCH}_{2} \mathrm{O}\right), 3.93$ (d, $2 \mathrm{H}, \mathrm{CH}_{2} \mathrm{OH}$ ), 4.46 (d with fine coupling, $4 \mathrm{H}, \mathrm{Ha}$, $\mathrm{Hb})$.

3-( $\omega$-Bromoalkyloxymethyl)-3-(5-bromopentyloxymethyl)oxetanes were similarly synthesized from 3-(5bromopentyloxymethyl)-3-hydroxymethyloxetane and 1, $\omega$-dibromoalkane.

3-(6-Bromohexyloxymethyl)-3-(5-bromopentyloxymethyl)oxetane.

Yield 39\%. $R_{\mathrm{f}}=0.35$ (hexane : ethyl acetate $=5: 1$ ).

${ }^{1} \mathrm{H}$ chemical shifts: $1.38-1.92\left(\mathrm{~m}, 14 \mathrm{H}, \mathrm{OCH}_{2}\left[\mathrm{CH}_{2}\right]_{3}\right.$ $\left.\mathrm{CH}_{2} \mathrm{O}, \mathrm{OCH}_{2}\left[\mathrm{CH}_{2}\right]_{4} \mathrm{CH}_{2} \mathrm{O}\right), 3.41$ and $3.42(\mathrm{t}, 4 \mathrm{H}$, $\left.J=6.0 \mathrm{~Hz}, \mathrm{CH}_{2} \mathrm{Br}\right), 3.46$ and $3.47(\mathrm{t}, 4 \mathrm{H}, J=6.0 \mathrm{~Hz}$, $\mathrm{CCH}_{2} \mathrm{OCH}_{2}$ ), 3.61 and $3.6\left(\mathrm{~s}, 4 \mathrm{H}, \mathrm{CCH}_{2} \mathrm{O}\right), 4.46(\mathrm{~s}, 4 \mathrm{H}$, $\mathrm{Ha}, \mathrm{Hb})$.

3-(7-Bromoheptyloxymethyl)-3-(5-bromopentyloxymethyl)oxetane.

Yield $28 \% . R_{\mathrm{f}}=0.30$ (hexane : ethyl acetate $=5: 1$ ).

${ }^{1} \mathrm{H}$ chemical shifts: $1.38-1.92\left(\mathrm{~m}, 16 \mathrm{H}, \mathrm{OCH}_{2}\left[\mathrm{CH}_{2}\right]_{3}\right.$ $\left.\mathrm{CH}_{2} \mathrm{O}, \mathrm{OCH}_{2}\left[\mathrm{CH}_{2}\right]_{5} \mathrm{CH}_{2} \mathrm{O}\right), 3.41$ and $3.42(\mathrm{t}, 4 \mathrm{H}$, $\left.J=6.0 \mathrm{~Hz}, \mathrm{CH}_{2} \mathrm{Br}\right), 3.46$ and $3.47(\mathrm{t}, 4 \mathrm{H}, J=6.0 \mathrm{~Hz}$, $\mathrm{CCH}_{2} \mathrm{OCH}_{2}$ ), 3.61 and $3.62\left(\mathrm{~s}, 4 \mathrm{H}, \mathrm{CCH}_{2} \mathrm{O}\right), 4.46(\mathrm{~s}, 4 \mathrm{H}$, $\mathrm{Ha}, \mathrm{Hb}$ ).

3,3-Bis \{5-(4-cyanobiphenyl-4'-yloxy)pentyloxymethyl oxetane $(O X-C N-5, C N-5)$. To a suspension of sodium hydride $(1.52 \mathrm{~g}, 38.0 \mathrm{mmol})$ in DMF $(10 \mathrm{~mL})$ was added dropwise DMF $(20 \mathrm{~mL})$ solution of 4-cyano-4'hydroxybiphenyl $(6.44 \mathrm{~g}, 33.0 \mathrm{mmol})$. After stirred for $30 \mathrm{~min}$, DMF $(15 \mathrm{~mL})$ solution of 3,3-bis(5-bromopentoxy)methyl)oxetane $(6.21 \mathrm{~g}, 15 \mathrm{mmol})$ was added and stirring was continued overnight at room temperature. Usual work up and removal of solvent left a crude oil, which was subjected to silica gel column chromatography (eluent, hexane: chloroform: diethyl ether $=2: 1: 1$ ) to give the product as a light yellow solid.

Yield $72 \% . \mathrm{mp} 34.7^{\circ} \mathrm{C} . R_{\mathrm{f}}=0.30$ (hexane : chloroform : diethyl ether $=1: 1: 2$ ).

${ }^{1} \mathrm{H}$ chemical shifts: $1.51-1.85\left(\mathrm{~m}, 12 \mathrm{H}, \mathrm{OCH}_{2}\left[\mathrm{CH}_{2}\right]_{3}{ }^{-}\right.$ $\left.\mathrm{CH}_{2} \mathrm{O}\right), 3.50\left(\mathrm{t}, 4 \mathrm{H}, \mathrm{J}=6.0 \mathrm{~Hz}, \mathrm{CCH}_{2} \mathrm{OCH}_{2}\right), 3.62(\mathrm{~s}$, $4 \mathrm{H}, \mathrm{CCH}_{2} \mathrm{O}$ ), $4.00\left(\mathrm{t}, 4 \mathrm{H}, \mathrm{J}=6.0 \mathrm{~Hz}, \mathrm{CH}_{2} \mathrm{OAr}\right), 4.48$ (s, $4 \mathrm{H}, H \mathrm{a}, H \mathrm{~b}), 6.98(\mathrm{~d}, 2 \mathrm{H}, J=8.8 \mathrm{~Hz}, H \mathrm{c}), 7.51$ (d, $2 \mathrm{H}$, $J=8.8 \mathrm{~Hz}, H \mathrm{~d}$ ), 7.65 (two d with fine coupling, $4 \mathrm{H}$, $J=7.8 \mathrm{~Hz}, H \mathrm{e}, H \mathrm{f})$.

3-(5-Bromopentyloxymethyl)-3-\{5-(4'-cyanobiphenyl4-yloxy)pentyloxymethyl $\}$ oxetane. This compound was similarly synthesized to OX-CN-5, CN- 5 by controlling the molar ratio of 4-cyano-4'-hydroxybiphenyl and 3,3bis(5-pentyloxymethyl)oxetane as $1: 2$.

Yield $52 \% . \quad R_{\mathrm{f}}=0.45$ (hexane : chloroform : diethyl ether $=1: 1: 2$ ).

${ }^{1} \mathrm{H}$ chemical shifts: $1.50-1.86\left(\mathrm{~m}, 12 \mathrm{H}, \mathrm{OCH}_{2}-\right.$ $\left.\left[\mathrm{CH}_{2}\right]_{3} \mathrm{CH}_{2}\right), 3.40\left(\mathrm{t}, 2 \mathrm{H}, \mathrm{J}=6.0 \mathrm{~Hz}, \mathrm{CH}_{2} \mathrm{Br}\right), 3.47(\mathrm{t}$, $\left.2 \mathrm{H}, J=6.0 \mathrm{~Hz}, \mathrm{OCH}_{2}\left[\mathrm{CH}_{2}\right]_{4} \mathrm{Br}\right), 3.51(\mathrm{t}, 2 \mathrm{H}, J=6.0 \mathrm{~Hz}$, $\left.\mathrm{OCH}_{2}\left[\mathrm{CH}_{2}\right]_{4} \mathrm{O}\right), 3.61\left(\mathrm{~s}, 2 \mathrm{H}, \mathrm{CCH}_{2} \mathrm{O}\right), 3.62(\mathrm{~s}, 2 \mathrm{H}$, $\left.\mathrm{CCH}_{2} \mathrm{O}\right), 4.02\left(\mathrm{t}, 2 \mathrm{H}, \mathrm{J}=6.0 \mathrm{~Hz}, \mathrm{CH}_{2} \mathrm{OAr}\right), 4.47$ (s, $4 \mathrm{H}$, $H \mathrm{a}, H \mathrm{~b}), 6.99(\mathrm{~d}, 2 \mathrm{H}, J=8.8 \mathrm{~Hz}, H \mathrm{c}), 7.53$ (d, $2 \mathrm{H}$, $J=8.8 \mathrm{~Hz}, H \mathrm{~d}$ ), 7.66 (two d with fine coupling, $4 \mathrm{H}$, 
$J=7.8 \mathrm{~Hz}, \mathrm{He}, H \mathrm{f})$.

$3-\{\omega$-(4'-Cyanobiphenyl-4-yloxy)alkyloxymethyl $\}-3-$ $\left\{5-\left(4^{\prime}\right.\right.$-cyanobiphenyl-4-yloxy)pentyloxy-methyl $\}$ oxetane (OX-CN-5, CN-m)s were similarly synthesized from 3( $\omega$-bromoalkyloxy-methyl)-3-(5-bromopentyloxymethyl)oxetanes.

3-\{6-(4'-Cyanobiphenyl-4-yloxy)hexyloxymethyl $\}-3-$ $\left\{5-\left(4^{\prime}\right.\right.$-cyanobiphenyl-4-yloxy)-pentyloxymethyl $\}$ oxetane $(\mathrm{OX}-\mathrm{CN}-5, \mathrm{CN}-6)$.

Yield $67 \% . \mathrm{mp} 55.7^{\circ} \mathrm{C} . \quad R_{\mathrm{f}}=0.30$ (hexane : ethyl acetate $=5: 1)$.

3-\{5-(4'-Cyanobiphenyl-4-yloxy)pentyloxymethyl $\}-3-$ $\left\{7-\left(4^{\prime}\right.\right.$-cyanobiphenyl-4-yloxy)-heptyloxymethyl $\}$ oxetane $(\mathrm{OX}-\mathrm{CN}-5, \mathrm{CN}-7)$.

Yield $52 \% . \mathrm{mp} 43.6^{\circ} \mathrm{C} . \quad R_{\mathrm{f}}=0.30$ (hexane: ethyl acetate $=5: 1$ ).

${ }^{1} \mathrm{H}$ chemical shifts $(\delta)$ of these compounds were observed at reasonable positions.

4-Methoxyphenyl 4-(hydroxy)benzoate was synthesized as reported (yield: $\left.92 \%, \mathrm{mp} 189^{\circ} \mathrm{C}\right) .{ }^{17,18)}$

3,3-Bis [5-\{4-(4'-methoxyphenyloxycarbonyl $)$ phenyloxy\}pentyloxymethyl]oxetane $(O X-1 B-5,1 B-5)$. The reaction of 3,3-bis(5-bromopentyloxymethyl)oxetane (4.97 g, $12 \mathrm{mmol}$ ) and 4-methoxyphenyl 4-hydroxybenzoate $(4.88 \mathrm{~g}, 20 \mathrm{mmol})$ in the presence of $\mathrm{K}_{2} \mathrm{CO}_{3}(3.31 \mathrm{~g}$, $24 \mathrm{mmol})$ in ethanol $(40 \mathrm{~mL})$ under reflux for $24 \mathrm{~h}$ gave the product after removal of the solvent and extraction with chloroform $(100 \mathrm{~mL})$. Silica gel column chromatography (eluent, hexane: chloroform: diethyl ether = $2: 1: 1)$ gave the pure product as a light yellow solid.

Yield $72 \% . \mathrm{mp} 66.2^{\circ} \mathrm{C} . R_{\mathrm{f}}=0.40$ (hexane : chloroform : diethyl ether $=1: 1: 2$ ).

${ }^{1} \mathrm{H}$ chemical shifts: $1.55-1.87\left(\mathrm{~m}, 12 \mathrm{H}, \mathrm{OCH}_{2}\left[\mathrm{CH}_{2}\right]_{3^{-}}\right.$ $\left.\mathrm{CH}_{2} \mathrm{O}\right), 3.50\left(\mathrm{t}, 4 \mathrm{H}, \mathrm{J}=6.3 \mathrm{~Hz}, \mathrm{CCH}_{2} \mathrm{OCH}_{2}\right), 3.63(\mathrm{~s}, 4 \mathrm{H}$, $\left.\mathrm{CCH}_{2} \mathrm{O}\right), 3.82\left(\mathrm{~s}, 6 \mathrm{H}, \mathrm{OCH}_{3}\right), 4.04(\mathrm{t}, 4 \mathrm{H}, J=6.3 \mathrm{~Hz}$, $\mathrm{CH}_{2} \mathrm{OAr}$ ), 4.48 (s, 4H, $\mathrm{Ha}, H \mathrm{~b}$ ), 6.94 (two d, 8H, $J=7.8 \mathrm{~Hz}, H \mathrm{~g}, H \mathrm{k}), 7.10(\mathrm{~d}, 4 \mathrm{H}, J=9.3 \mathrm{~Hz}, H \mathrm{j}), 8.12$ (d, $4 \mathrm{H}, J=9.0 \mathrm{~Hz}, H \mathrm{i})$.

3-\{5-(4'-Cyanobiphenyl-4-yloxy)pentyloxymethyl $\}-3-$ [5-\{4-(4'-methoxyphenyloxycarbonyl)-phenyloxy $\}$ pentyloxymethyl]oxetane $(O X-C N-5,1 B-5)$. This compound was synthesized from 3-(5-bromopentyloxymethyl)-3-\{5(4'-cyanobiphenyl-4-yloxy)pentyloxymethyl $\}$ oxetane (4.24 g, $8 \mathrm{mmol}$ ) and 4-methoxyphenyl 4-hydroxybenzoate $(1.95 \mathrm{~g}, 8 \mathrm{mmol})$ in the presence of potassium carbonate.

Yield $56 \% \cdot \mathrm{mp} 48.1^{\circ} \mathrm{C} . R_{\mathrm{f}}=0.40$ (hexane : chloroform : diethyl ether $=1: 1: 2$ ).

${ }^{1} \mathrm{H}$ chemical shifts: $1.55-1.87\left(\mathrm{~m}, 12 \mathrm{H}, \mathrm{OCH}_{2}\left(\mathrm{CH}_{2}\right)_{3}-\right.$ $\left.\mathrm{CH}_{2} \mathrm{O}\right), 3.50\left(\mathrm{t}, 4 \mathrm{H}, \mathrm{J}=6.3 \mathrm{~Hz}, \mathrm{CCH}_{2} \mathrm{OCH}_{2}\right), 3.63(\mathrm{~s}, 4 \mathrm{H}$, $\left.\mathrm{CCH}_{2} \mathrm{O}\right), 3.82\left(\mathrm{~s}, 6 \mathrm{H}, \mathrm{CH}_{3}\right), 4.04(\mathrm{t}, 4 \mathrm{H}, J=6.3 \mathrm{~Hz}$, $\mathrm{CH}_{2} \mathrm{OAr}$ ), 4.48 (s, 4H, $\mathrm{Ha}, \mathrm{Hb}$ ), 6.94 (two d, 8H, $J=7.8 \mathrm{~Hz}, H \mathrm{~g}, H \mathrm{k}), 7.10(\mathrm{~d}, 4 \mathrm{H}, J=9.3 \mathrm{~Hz}, H \mathrm{j}), 8.12(\mathrm{~d}$, $4 \mathrm{H}, J=9.0 \mathrm{~Hz}, \mathrm{Hi})$.

Similarly 3-\{5-(4'-Cyanobiphenyl-4-yloxy)pentyloxymethyl $\}-3-\left[5-\left\{4-\left(4^{\prime}\right.\right.\right.$-decyloxyphenyloxycarbonyl)phenyloxy\}pentyloxymethyl]oxetane (OX-CN-5, 10B-5) was synthesized.

\section{Polymerization}

Dichloromethane was dried over calcium hydride. Monomers were dried over calcium hydride as a solution in dichloromethane, and used as the solution after fil- tration of calcium hydride. Polymerizations were carried out in dichloromethane for $24-48 \mathrm{~h}$ at $0^{\circ} \mathrm{C}$. Triethyloxonium tetrafluoroborate $\left(1.0 \mathrm{~mol} \mathrm{~L}^{-1}\right)$ was used as an initiator. Polymers were recovered by pouring in methanol and purified by repeated reprecipitation from dichloromethane into methanol or ethanol.

\section{$P O X-C N-5, C N-5$.}

Yield $21 \%$.

${ }^{1} \mathrm{H}$ chemical shifts: $1.38-1.87\left(\mathrm{br}, 12 \mathrm{H}, \mathrm{OCH}_{2}\left(\mathrm{CH}_{2}\right)_{3}-\right.$ $\mathrm{CH}_{2} \mathrm{O}$ ), 3.27-3.51 (br, 8H, $\mathrm{CCH}_{2} \mathrm{OCH}_{2}$ and main chain proton), 3.69-3.79 (br, $4 \mathrm{H}, \mathrm{CCH}_{2} \mathrm{OCH}_{2}$ ), 3.85-3.92 (br, 4H, CH $\mathrm{H}_{2} \mathrm{OAr}$ ), 6.82-6.92 (br, 4H, Hc), 7.41-7.72 (br m, $12 \mathrm{H}, \mathrm{Hd}, \mathrm{He}, H \mathrm{f}$ ).

${ }^{13} \mathrm{C}$ chemical shifts: 22.36 (C6), 28.43 (C5), 28.63 (C7), $45.26(\mathrm{C} 2), 67.69(\mathrm{C} 1), 69.64$ and 70.87 (C3, C4, and C8), 109.64 (C16), 114.02 (C10), 118.36 (C17), 126.55 (C14), 127.86 (C11), 131.68 (C12), 132.11 (C15), 144.64 (C13), 159.24 (C9).

$P O X-1 B-5,1 B-5$.

Yield $46 \%$

${ }^{1} \mathrm{H}$ chemical shifts: $1.46-1.88\left(\mathrm{br}, 12 \mathrm{H}, \mathrm{OCH}_{2}\left(\mathrm{CH}_{2}\right)_{3}\right.$ $\mathrm{CH}_{2} \mathrm{O}$ ), 3.29-3.46 (br, $8 \mathrm{H}, \mathrm{CCH}_{2} \mathrm{OCH}_{2}$ and main chain proton), 3.72 (brs, 4H, $\mathrm{CCH}_{2} \mathrm{OCH}_{2}$ ), 3.79 (br s, 6H, $\mathrm{OCH}_{3}$ ), 3.89-4.03 (br, 4H, $\left.\mathrm{CH}_{2} \mathrm{OAr}\right), 6.82-6.94$ (br m, $8 \mathrm{H}, H \mathrm{~g}, H \mathrm{j}), 6.99-7.12(\mathrm{br}, 4 \mathrm{H}, H \mathrm{i}), 8.02-8.13$ (br, $4 \mathrm{H}, H \mathrm{~h})$.

${ }^{13} \mathrm{C}$ chemical shifts: 22.37 (C6), 28.54 (C5), 28.98 (C7), 45.42 (C2), 55.15 (C18), $67.38(\mathrm{C} 1), 69.08$ and $70.80(\mathrm{C} 3$, C4, and C8), 113.72 (C10), 114.55 (C16), 121.31 (C12), 122.02 (C15), 131.74 (C11), 144.03 (C14), 156.77 (C17), 162.87 (C13), 164.92 (C9).

$P O X-C N-5,1 B-5$.

Yield $51 \%$.

${ }^{1} \mathrm{H}$ chemical shifts: $1.44-1.88\left(\mathrm{br}, 12 \mathrm{H}, \mathrm{OCH}_{2}\left(\mathrm{CH}_{2}\right)_{3^{-}}\right.$ $\left.\mathrm{CH}_{2} \mathrm{O}\right), 3.33-3.45$ (br, $8 \mathrm{H}, \mathrm{CCH}_{2} \mathrm{OCH}_{2}$ and main chain proton), $3.89-4.03$ (br m, $11 \mathrm{H}, \mathrm{CCH}_{2} \mathrm{OCH}_{2}, \mathrm{CH}_{2} \mathrm{OAr}$, and $\left.\mathrm{OCH}_{3}\right), 6.82-7.08$ (brm, $8 \mathrm{H}, \mathrm{Hg}, \mathrm{Hi}, \mathrm{Hj}$ ), $7.38-7.69$ (br, 6H, $H \mathrm{~d}, H \mathrm{e}, H \mathrm{f}), 8.02-8.13(\mathrm{br}, 2 \mathrm{H}, H \mathrm{~h})$. $P O X-C N-5, C N-6$.

Yield: $23 \%$.

POX-CN-5,CN-7.

Yield $17 \%$.

These polymers also gave reasonable NMR analytical data.

\section{RESULTS AND DISCUSSION}

\section{Synthesis}

The introduction of spacer groups by the formation of ether linkages from 3,3-bis(hydroxymethyl)oxetane was achieved in good yield by the use of phase transfer catalyst or sodium hydride. Selective introduction of only one cyanobiphenyl group to 3,3-bis $\{$ (5-bromopentyloxy)methyl $\}$ oxetane was possible in moderate $(52 \%)$ yield by using excess 3,3-bis $\{$ (5-bromopentyloxy)methyl $\}$ oxetane to 4-cyano-4'-hydroxybiphenyl. Extent of reaction was estimated by ${ }^{1} \mathrm{H}$ NMR by the peak at $3.4 \mathrm{ppm}$ (BrCH $\mathrm{CH}_{2}$ : disappearing) and $4.0 \mathrm{ppm}\left(\mathrm{OCH}_{2}\right.$ : appearing). Introduction of only one spacer group by mono alkylation of 3,3-bis(hydroxymethyl)oxetane by 1, $\omega$-dibromoalkane was possible by using excess 3,3-bis(hydroxymethyl)oxetane to sodium hydride in moderate to rather poor $(22 \%)$ yield. 
Table I. Polymerization of oxetane monomers ${ }^{\mathbf{a}}$

\begin{tabular}{|c|c|c|c|c|c|c|}
\hline Monomer & $\frac{\text { Concn. }}{\mathrm{mol} \mathrm{1}^{-1}}$ & $\frac{\text { Initiator }}{\operatorname{mol} \%}$ & $\frac{\text { Yield }}{\%}$ & $M_{n}^{\mathrm{b}} \times 10^{-3}$ & $M_{w}{ }^{\mathrm{b}} \times 10^{-3}$ & $M_{w} / M_{n}^{\mathrm{b}}$ \\
\hline OX-CN-5, CN-5 & 1.00 & $\mathrm{Et}_{3} \mathrm{O}^{+} \mathrm{BF}_{4}^{-}(1.00)$ & 21 & 4.5 & 11.0 & 2.3 \\
\hline OX-CN-5, 1B-5 & 1.00 & $\mathrm{Et}_{3} \mathrm{O}^{+} \mathrm{BF}_{4}^{-}(1.00)$ & 46 & 5.0 & 11.0 & 2.2 \\
\hline OX-1B-5, 1B-5 & 1.00 & $\mathrm{Et}_{3} \mathrm{O}^{+} \mathrm{BF}_{4}^{-}(1.00)$ & 51 & 4.6 & 12.0 & 2.6 \\
\hline OX-CN-5, CN-6 & 1.00 & $\mathrm{Et}_{3} \mathrm{O}^{+} \mathrm{BF}_{4}^{-}(1.00)$ & 23 & 6.7 & 11.0 & 1.6 \\
\hline OX-CN-5, CN-5 & 1.00 & $\mathrm{Et}_{3} \mathrm{O}^{+} \mathrm{BF}_{4}^{-}(2.00)$ & 8 & 2.5 & 4.3 & 1.6 \\
\hline OX-CN-5, 1B-5 & 1.00 & $\mathrm{Et}_{3} \mathrm{O}^{+} \mathrm{BF}_{4}^{-}(2.00)$ & 10 & 3.6 & 5.4 & 1.5 \\
\hline $\mathrm{OX}-\mathrm{CN}-5, \mathrm{CN}-7$ & 1.00 & $\mathrm{Et}_{3} \mathrm{O}^{+} \mathrm{BF}_{4}^{-}(2.00)$ & 18 & 2.8 & 4.1 & 1.5 \\
\hline $\mathrm{OX}-\mathrm{CN}-5^{\mathrm{c}}$ & 1.00 & $\mathrm{BF}_{3} \mathrm{OEt}_{2}$ & 34 & 13.0 & 23.0 & 1.8 \\
\hline
\end{tabular}

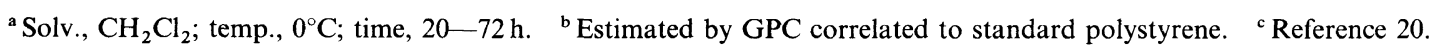

Table II. Thermal behavior of monomers

\begin{tabular}{|c|c|c|c|}
\hline \multirow[t]{2}{*}{ Monomer } & \multicolumn{2}{|c|}{ Transition temp $\left({ }^{\circ} \mathrm{C}\right)^{\mathrm{a}}$ and enthalpy $\left(\mathrm{kJ} \mathrm{mol}^{-1}\right)$} & \multirow[t]{2}{*}{ Texture } \\
\hline & Heating & Cooling & \\
\hline OX-CN-5, 1B-5 & 52.2 (broad) (0.42) & 50.6 (broad) $(0.42)$ & Schlieren \\
\hline $\mathrm{OX}-\mathrm{CN}-5,10 \mathrm{~B}-5$ & $64.1(2.71)$ & $63.6(2.21)$ & Fan \\
\hline OX-1B-5, 1B-5 & 24.3 (broad) $(2.7), 69.3 ; 77.1$ (broad) $(3.3)$ & $21.8 ; 23.8(2.7), 61.3 ; 73.1(3.3)$ & Schlieren \\
\hline $\mathrm{OX}-\mathrm{CN}-5, \mathrm{CN}-6$ & $50.2,63.3(1.05)$ & $50.0,56.9(1.05)$ & Schlieren \\
\hline $\mathrm{OX}-\mathrm{CN}-5, \mathrm{CN}-7$ & $45.0(0.61)$ & $45.0(0.61)$ & Schlieren \\
\hline
\end{tabular}

${ }^{a}$ Determined by DSC on 2 nd heating and cooling. Temperature at peak.

\section{Polymerization}

The results of the polymerization are summarized in Table I.

The ring-opening polymerization of monomers with cyano group is very slow. ${ }^{14}$ First, polymerizations were carried out with $2 \mathrm{~mol} \%$ initiator. However, the yield was low and much of the low molecular weight fractions was formed. Since the molecular weight of produced polymers was about 3000 , the yield also decreased due to the difficulty to separate the polymers from unreacted monomers. The concentration of the initiator was decreased to $1.0 \mathrm{~mol} \%$ to suppress the formation of oligomers. Molecular weight was increased, but molecular weight distribution became bimodal. It was suggested by Rose ${ }^{4,46}$ that the cyclic oligomers are formed in the cationic polymerization of oxetane. Some cyclic oligomers may have been formed in these systems.

In the polymerization of OX-1B-5, 1B-5, the formation of oligomers was not noted. The extent of cyclic oligomer formation depends on the chemical structure of monomer, especially, on the substituent on mesogenic group. The polymers were purified by removing the oligomers by reprecipitation or by silica gel column chromatography (eluent: chloroform). Low molecular weight fraction seen in the GPC chromatogram before purification has been removed by these treatment. The absence of monomer in polymer was checked by GPC.

\section{Thermal Analysis}

DSC analysis of monomers was summarized in Table

We reported that monomers with only one mesogenic group (OX-CN-m) did not show liquid crystalline phase. ${ }^{14} \mathrm{OX}-\mathrm{CN}-5, \mathrm{CN}-5$ and $\mathrm{OX}-\mathrm{CN}-5,1 \mathrm{~B}-5$ have transition temperatures at $39.0^{\circ} \mathrm{C}$ and at $52.2^{\circ} \mathrm{C}$ in heating cycle. Optical polarized micrographs showed that these monomers formed schlieren texture presumably indicating a nematic phase. OX-1B-5, 1B-5 showed four transition peaks. Under optical polarized microscopy at $54.2^{\circ} \mathrm{C}$ in cooling cycle, schlieren texture was observed for a short time and soon the compound solidified at $40.8^{\circ} \mathrm{C}$. OX-CN-5, CN-6 and OX-CN-5, CN-7 showed two transition temperatures. Optical polarization micrographs revealed that these compounds took presumably the nematic phase suggested by schlieren texture. Thus, all these monomers were considered to take the nematic liquid crystalline phase. This is apparently because the orientation of monomers by overcoming the steric hindrance of oxetane ring became possible by linking two mesogenic group.

By making tail group longer, OX-CN-5, 10B-5 showed a fan structure when annealed at $63.3^{\circ} \mathrm{C}$. The structure of tail group is also important in determining the liquid crystalline structure. The actual arrangement of monomer molecules in the liquid crystalline state should be elucidated by X-ray diffraction analysis. III.

DSC analysis of polymers was summarized in Table

Contrary to all monomers except OX-CN-5, 10B-5 took the nematic phase, all polymers except POX1B-5, 1B-5 took the smectic phase. The transition temperature from smectic liquid crystalline state to isotropic phase of POX-CN-5, CN-5 is observed at $137.5^{\circ} \mathrm{C}$, higher than that of polyoxetane having one cyanobiphenyl group POX-CN-5, whose transition temperature was observed at $96^{\circ} \mathrm{C} .{ }^{14}$ The glass transition temperatures remained 
Table III. Thermal behavior of poly(oxetane)s

\begin{tabular}{cccc} 
& \multicolumn{2}{c}{ Transition temp $/{ }^{\circ} \mathrm{C}^{\mathrm{a}}$} & \\
\cline { 2 - 3 } Polymers & $T_{\mathrm{g}}^{\mathrm{b}}$ & $T_{\mathrm{i}}$ (enthalpy, $\left.\mathrm{Jg}^{-1}\right)^{\mathrm{c}}$ & \\
\hline POX-CN-5, CN-5 & 24.4 & $137.5(2.4)$ & Fan \\
POX-CN-5, 1B-5 & 18.2 & $136.3(3.0)$ & Fan \\
POX-1B-5, 1B-5 & n.d. $^{\mathrm{d}}$ & $109.9(1.5)$, & Schlieren \\
& & $120.8(2.3)$ & \\
POX-CN-5, CN-6 & 20.6 & $138.4(2.3)$ & Fan \\
POX-CN-5, CN-7 & 14.7 & $122.6(3.3)$ & Fan
\end{tabular}

${ }^{a}$ Determined by DSC. ${ }^{\mathrm{b}}$ Temperature where transition starts on 2 nd heating. ${ }^{\mathrm{c}}$ Temperature at peak on 2 nd heating. ${ }^{\mathrm{d}}$ Not determined.

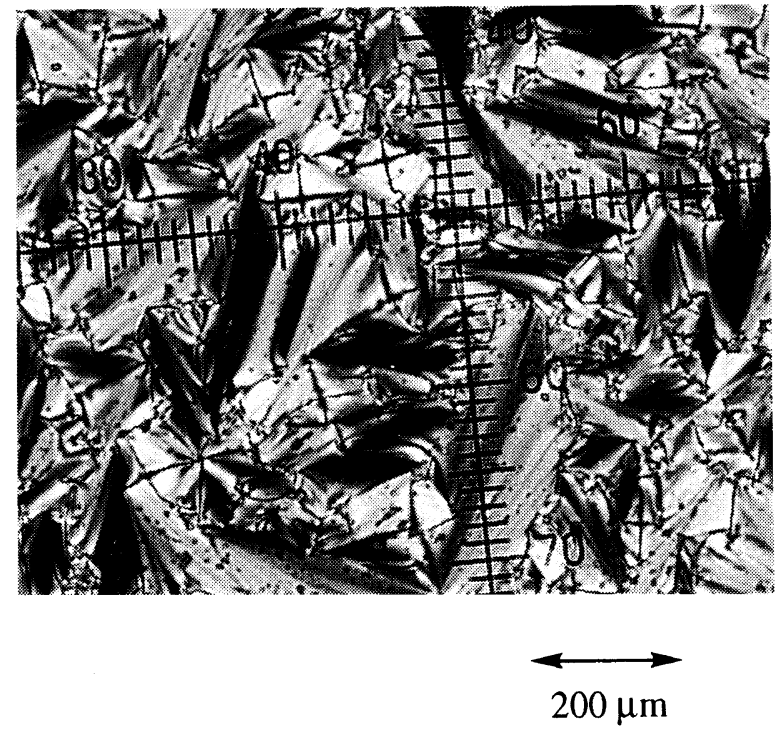

Figure 2. Optical polarized micrograph of POX-CN-5, CN-7.

almost the same. Accordingly, by the introduction of second cyanobiphenyl mesogenic groups in the repeating unit, the liquid crystalline temperature range became wider.

POX-1B-5, 1B-5 showed two transition temperatures, and when annealed at $118.8^{\circ} \mathrm{C}$, schlieren texture was observed indicating the nematic phase. Apparently the two benzoate mesogenic groups in the repeating unit are organized not well enough to take the smectic phase. POX-CN-5, 1B-5 showed the smectic phase. Orientation of cyanobiphenyl was not so much affected by benzoate. The difference in the length of the two spacers may create the inclination of mesogenic groups. Both POX-CN-5, CN-6 and POX-CN-5, CN-7 showed only one transition temperature. POX-CN-5, CN-6 showed focal-conic fan texture, indicating smectic A phase. The texture of
POX-CN-5, CN-7 shown in Figure 2 may indicate a different phase other than the smectic A.

\section{CONCLUSION}

The presence of two cyano groups in the monomer strongly suppressed the polymerizability of the oxetane monomer by $\mathrm{Et}_{3} \mathrm{O}^{+} \mathrm{BF}_{4}^{-}$. In contrast to all monomers except OX-CN-5, 10B-5 took the nematic, polymers took the smectic phase except POX-1B-5, 1B-5. The liquid crystalline temperature became higher and the temperature range became wider by the presence of a second mesogenic group.

Acknowledgment. This work was partially supported by a Grant in Aid for Scientific Research (08455438) and a Grant in Aid for Scientific Research in Priority Areas, "New Polymers and Their Nano-Organized Systems" (08246223) from the Ministry of Education, Science, Sports, and Culture of Japan.

\section{REFERENCES}

1, Y. Kawakami, M. Suzuki, Y. Kato, and A. Mori, Polym. J., 28, 845 (1996).

2. N. A. Plate, V. P. Shivaev (translated by S. L. Schnur, J. M. G. Cowie), "Comb-Shaped Polymers and Liquid Crystals," Plenum, New York, N.Y., (1987).

3. K. Yonetake, M. Nakagomi, M. Ueda, and T. Masuko, Polym. J., 29, 240 (1997).

4. G. Ping and C. Chang, private communication.

5. K. Sugiyama and K. Shiraishi, Bull. Chem. Soc. Jpn., 64, 1715 (1991).

6. C. T. Imrie, F. E. Karasz, and G. S. Attard, Macromolecules, 25, 1278 (1992).

7. C. T. Imrie, F. E. Karasz, and G. S. Attard, Macromolecules, 27, 1578 (1994).

8. S. Ujiie, Y. Tanaka, and K. Iimura, Chem Lett., 1037 (1991).

9. Y. Kosaka and T. Uryu, Macromolecules, 28, 870 (1995).

10. M. Yamada, T. Iguchi, A. Hirano, S. Nakahama, and J. Watanabe, Macremelecules, 28, 50 (1995).

11. S. Diele, S. Oelsner, F. Kusche, B. Hisgen, H. Ringsdorf, and R. Zentel, Makromol. Chem., 188, 1993 (1987).

12. S. Diele, B. Hisgen, B. Reck, and H. Ringsdorf, Makromol. Chem., Rapid Commun., 7, 267 (1986).

13. Y. K. Han, D. Y. Kim, and Y. K. Kim, J. Polym. Sci. Part A, 30, 1177 (1992).

14. Y. Kawakami, K. Takahashi, S. Nishiguchi, and K. Toida, Polym. Int., 31, 35 (1993).

15. Y. Kawakami, M. Suzuki, Y. Kato, and A. Mori, Polym. J., 28, 845 (1996).

16. M. Motoi, H. Suda, K. Kijima, T. Doi, T. Nakagawa, and S. Kanoh, Polym. J., 21, 451 (1989).

17. P. A. Tuan, S. G. Kostromin, and V. P. Shibaev, Polym. Bull., 20, 249 (1993).

18. R. A. Hikmet, J. Lub, and A. J. W. Tol, Macromolecules, 28, 3313 (1995).

19. J. B. Rose, J. Chem. Soc., 542 (1956).

20. J. B. Rose, J. Chem. Soc., 546 (1956). 